\title{
Automatic boundary extraction and rectification of bony tissue in CT images using artificial intelligence techniques
}

\author{
Matthew F. Y. Kwan*, K. C. Cheung and Ian Gibson \\ Department of Mechanical Engineering, The University of Hong Kong \\ Pokfulam Road, Hong Kong
}

\begin{abstract}
A novel approach is presented for fully automated boundary extraction and rectification of bony tissue from planar CT data. The approach extracts and rectifies feature boundary in a hierarchical fashion. It consists of a fuzzy multilevel thresholding operation, followed by a small void cleanup procedure. Then a binary morphological boundary detector is applied to extract the boundary. However, defective boundaries and undesirable artifacts may still be present. Thus two innovative anatomical knowledge based algorithms are used to remove the undesired structures and refine the erroneous boundary. Results of applying the approach on lumbar CT images are presented, with a discussion of the potential for clinical application of the approach.
\end{abstract}

Keywords: boundary extraction, boundary rectification, CT, artificial intelligence, thresholding, reconstruction

\section{INTRODUCTION}

Three-dimensional medical models are increasingly important in diagnosis and surgery planning. They are usually reconstructed from two-dimensional medical image data such as CT and MRI scans. To this end, they require some form of tissue classification, or more generally, image segmentation and boundary detection. The set of boundaries of an anatomical feature serves as the input for later 3D surface or solid model reconstruction' ${ }^{1}$. In CT images, feature boundaries obtained by conventional convolution-based edge detection algorithms, such as Sobel, Robert, Canny and Kirsch detectors, are often unsatisfactory due to partial volume effect and noise effect. Though many adaptive edge tracing techniques have been developed ${ }^{2-4}$, a certain amount of human intervention is often needed.

The segmentation problem for CT data is complicated. The solution depends on a large number of internal and external factors. Internal factors are related to the scanned specimen and refer to the inherent properties such as density, shape and geometric variance of the feature. External factors relate to the parameters of the scanning process such as image contrast, resolution, signal to noise ratio, slice thickness, data set size, and presence of artifacts. Owing to these factors, it is extremely difficult to develop a universal approach for all segmentation tasks without applying a priori knowledge. Also, for accurate volume reconstruction, small slice thicknesses are often required, which leads to large data sets. The need for higher levels of automation of the segmentation process grows with the size of the data set.

A range of approaches has been proposed for extracting structure boundaries with varying levels of automation and practical applicability. Barrett and Mortensen ${ }^{5}$ reported on an interactive boundary extraction technique, operating on individual slice images. Clarysse et al. ${ }^{6}$ designed an algorithm based on deformable model concept and a priori knowledge. Cheng et al. ${ }^{7}$ introduced fuzzy logic and relaxation techniques to extract boundaries for three-dimensional ultrasonic echo images. Hunter et al. ${ }^{8}$ proposed two fully automatic approaches using fuzzy logic and artificial neural networks in the extraction process.

In this paper we propose an automatic approach, operating on two-dimensional CT data sets, which aims to extract an accurate boundary of the bony tissue. This approach begins with multilevel thresholding to generate an initial binary image. The white region in the image is considered as the region of interest (ROI). A small void removal algorithm fills up the tiny holes inside the ROI. To eliminate artifacts, an artifact removal algorithm is used that applies anatomical knowledge. Contours are then traced out by a binary boundary detector. The erroneous boundary segments are rectified in a later step by a knowledge-based algorithm.

\footnotetext{
" Correspondence: Email: h9412180@hkusua.hku.hk; Telephone: (852) 2859 2824; Fax: (852) 28585415
} 


\section{METHODOLOGY}

The approach can be divided into five distinct functional steps: fuzzy multilevel thresholding, small void removal, binary morphological boundary detector, knowledge-based artifact removal and knowledge-based boundary rectification. Figure 1 shows a block diagram illustrating the approach for boundary extraction and rectification.

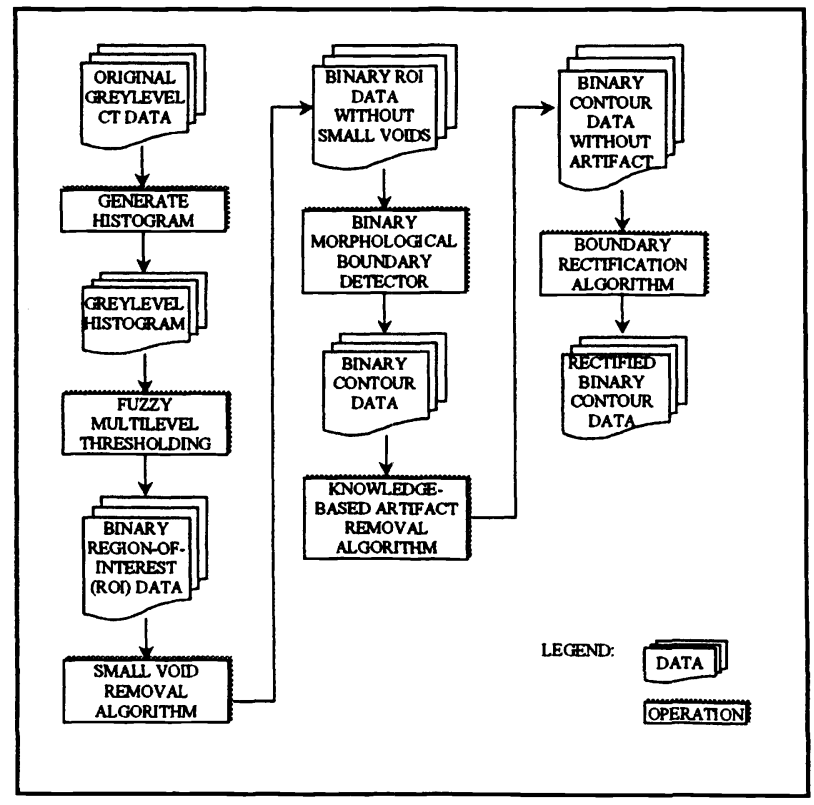

Figure 1. A schematic diagram of the proposed approach.

\subsection{Fuzzy Multilevel Thresholding}

A CT image contains pixels that are expressed in discrete grey levels. A histogram of grey levels can show characteristic intensity ranges for major anatomical classes including soft tissue, bony tissue, fat and empty space regardless of the presence of noise and artifacts. This is because the intensity shown depends on the spatial density of the scanned object. If an object is homogeneous and its density is distinct from the surrounding objects, its grey level would be obviously observable in the histogram. The lower the density, the darker the appearance. For example, bony tissue is always white whereas empty space is dark. It is impossible to represent an anatomical class by a unique grey level due to the heterogeneity of anatomical structures, presence of statistical noise and beam hardening effect. Instead, an intensity range can be used to represent that anatomical class. The range is not fixed for each class and a class range may be different for different scans. Hence, with the aid of a grey level histogram, a CT image can be modelled by a multi-modal histogram as shown in Figure 2. Each anatomical object can be approximated by a normal distribution curve with a peak and a certain standard deviation. Under this assumption, an intensity range for an object can be measured from the trough on the left of a peak to the trough on the right of that peak. 


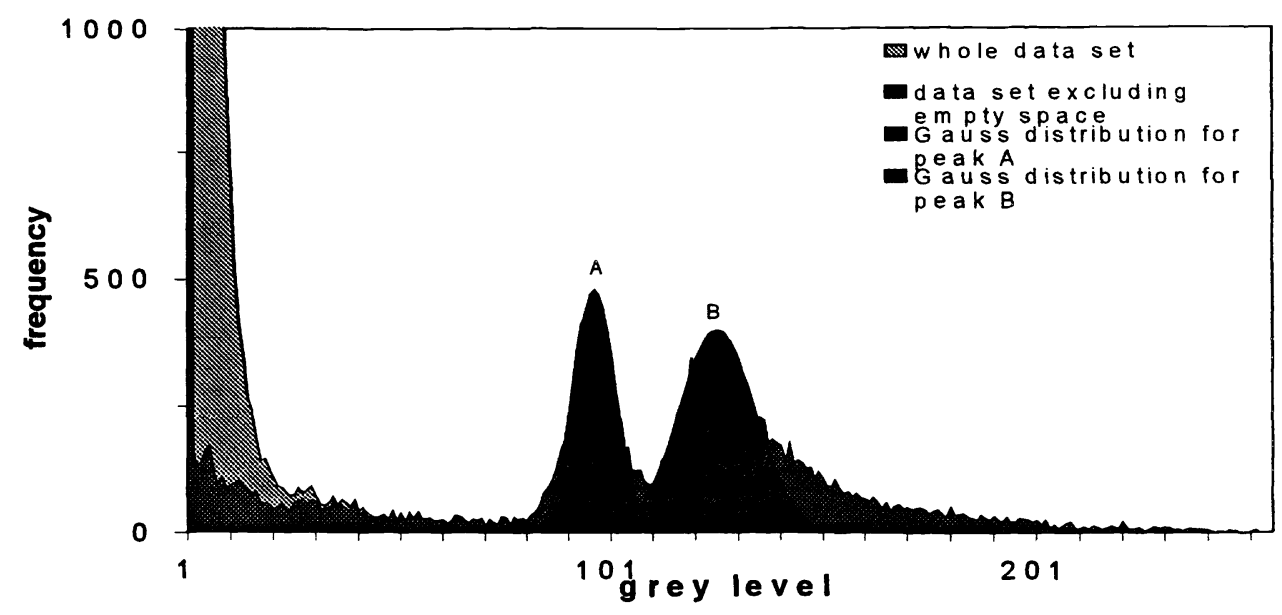

Figure 2. Grey level histogram showing the data set with the two peaks fitted by Gaussian distribution curves.

Two observations lead us to a thresholding approach for initial segmentation. Firstly, the bony tissue is quite homogeneous. Therefore, there is a good chance of finding a well-defined range in the grey level histogram to represent it. Secondly, regardless of the CT scan setting, the image intensity of the bony tissue is generally distinct from the other tissues. Therefore, a single range of grey levels can be used to represent the bone in a particular scan, and separate it from the other entities. This does not mean, however, that any such intensity range will represent exclusively the object.

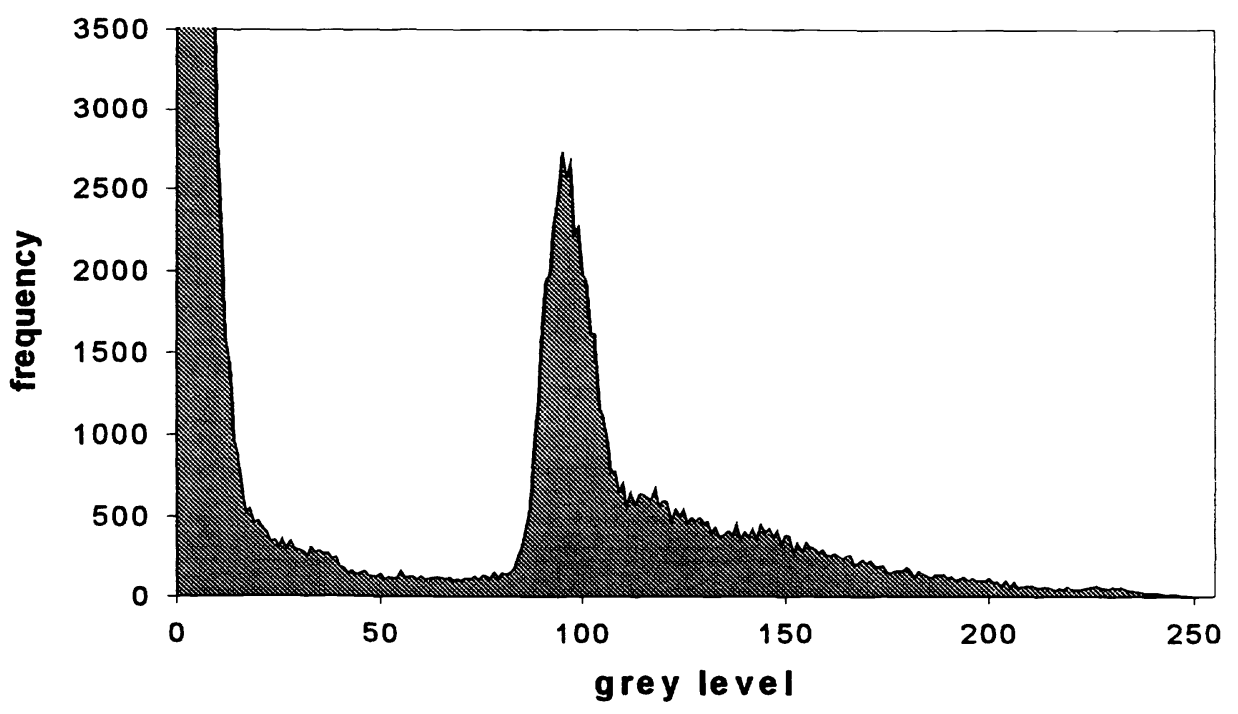

Figure 3. A grey level histogram without two conspicuously distinct peaks.

In general, the threshold is located at the obvious and deep valley of the histogram. Nevertheless, when the valley is not so obvious as shown in Figure 3, it is very difficult to determine the threshold. Fuzzy set theory has been applied to address this issue and obtained remarkable results ${ }^{9}$. It essentially partitions the image space into meaningful regions by minimizing the measure of fuzziness of the image. The measure is commonly expressed by entropy. As a CT scan contains more than two objects, multilevel thresholding is needed.

Consider the image $X$ as a set of size $\mathrm{M} \times \mathrm{N}$ with $\mathrm{L}$ grey levels and $x_{m n}$ is the grey level of the pixel located at $(\mathrm{m}, \mathrm{n})$. Let $\mu_{X}\left(x_{m n}\right)$ denote the membership value which represents the degree of belonging to a certain class by this pixel. $\mu_{X}\left(x_{m n}\right)$ is a real number bounded by $[0,1]$. In fuzzy set notation, the image set $X$ can be written as 


$$
X=\left\{\left(x_{m v}, \mu_{x}\left(x_{m n}\right)\right)\right\}
$$

where $0 \leq \mu_{X}\left(x_{m n}\right) \leq 1, m=0,1, \ldots, M-1$ and $n=0,1, \ldots, N-1$.

Let $h(g)$ be the frequency at grey level $g$. Suppose there are $K$ classes separated by $K-l$ thresholds $\left(\mathrm{T}_{1}, \mathrm{~T}_{2}, \ldots, \mathrm{T}_{K-1}\right)$, where $T_{1}<T_{2}<\ldots<T_{K-1}$. The average grey level of the $p^{\text {th }}$ class is:

$$
\mu_{p}=\frac{\sum_{g=T_{p}}^{T} g h(g)}{\sum_{g=T_{p}+1}^{T} h(g)}
$$

The average grey levels can be regarded as the target values of different classes for the given set of threshold values $\left(T_{1}\right.$, $\mathrm{T}_{2}, \ldots, \mathrm{T}_{K-1}$ ), The relationship between a pixel and its belonging region should depend on the absolute difference of its grey level and the target value of its belonging class. Hence, the membership function which evaluates this relationship for the pixel at location $(\mathrm{m}, \mathrm{n})$ can be defined as:

$$
\mu_{X}\left(x_{m n}\right)=\frac{1}{1+\left|x_{m n}-\mu_{p}\right| / C} \quad \text { if } \mathrm{T}_{\mathrm{p}} \leq x_{m n}<\mathrm{T}_{\mathrm{p}+1}
$$

where $C$ is a constant so chosen that $1 / 2 \leq \mu_{X}\left(x_{m n}\right) \leq 1$.

The entropy based on the Shannon's function is defined as:

$$
E\left(T_{1}, T_{2}, \ldots, T_{K-1}\right)=\frac{1}{\mathrm{MN} \ln 2} \sum_{\mathrm{g}} \mathrm{S}\left(\mu_{\mathrm{X}}(\mathrm{g})\right) \mathbf{h}(\mathrm{g}) \quad \mathrm{g}=0,1, \ldots, L-1
$$

with the Shannon's function given by:

$$
\mathrm{S}\left(\mu_{X}(g)\right)=-\mu_{X}(g) \ln \left[\mu_{X}(g)\right]-\left[1-\mu_{X}(g)\right] \ln / 1-\mu_{X}(g) /
$$

Determining the optimal threshold values for a given image is a heuristic process, that is, every possible combination of thresholds must be taken into account. If a threshold combination gives the lowest $\mathrm{E}$, it is most probably the best solution. Sometimes, the threshold found is not necessarily located at the deepest valley between two peaks. To remedy this, Huang and Wang ${ }^{10}$ suggested to find a better threshold within a predefined fuzzy range. Theoretically, the threshold should have a better chance of being located at the real valley and give better results. The result of this thresholding operation on a typical axial spine cross-sectional image is shown in Figure 4. Procedures for correcting misclassified regions are described in the following sections.

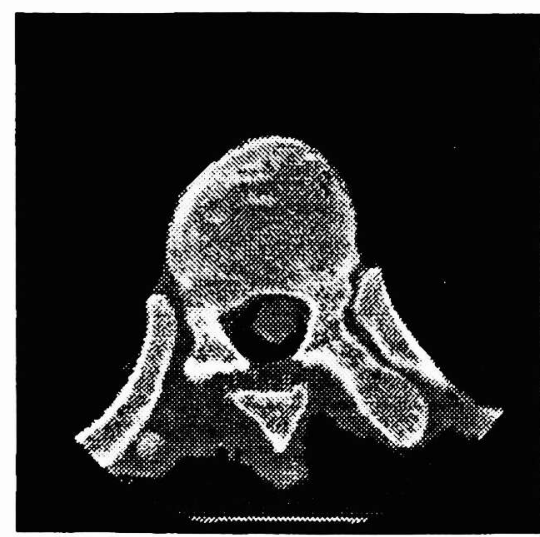

(a) Original image

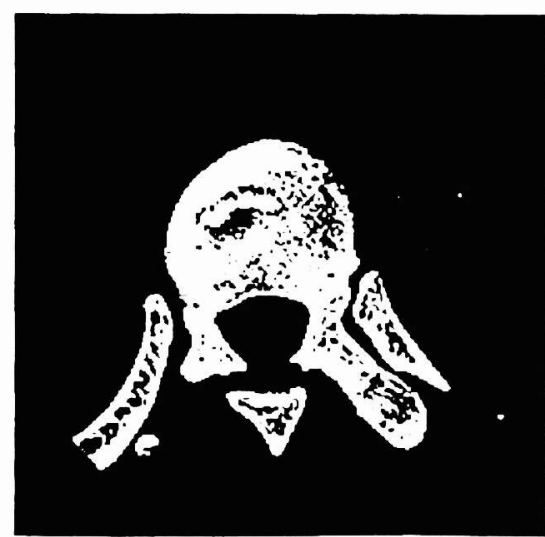

(b) Thresholded image

Figure 4. Original image and thresholded image. 


\subsection{Small Void Removal Algorithm}

Single-pixel objects in background and single-pixel holes in an object of a binary image, referred to as salt-and-pepper noise, are often the result of propagation of uncorrelated noise in the original image through some binarization process. They can be easily detected as their grey levels are different from their neighbours. They can be removed by changing their grey levels. However, small, insignificant holes of several pixels in size in the object or small noisy regions in the background are difficult to be eliminated. The proposed algorithm is based on a region-growing technique and attempts to get rid of small voids by checking the size of them. Suppose we have a binary image with both background and holes in black (label value $=0$ ) and object in white (label value $=1$ ). The first step is to separate the background and the holes into two groups by labelling the pixels with different values. We select a background pixel as a seed. Using this seed pixel, the neighbouring pixels will be checked whether they are background pixels. If a neighbour pixel has the same grey level as the seed pixel, it will be marked as " 2 " and form another seed. The process is called region-growing and continues until no more seed is detected. Now we have an image with three distinct groups: background (label value $=2$ ), objects (label value $=1$ ) and voids (label value $=0$ ). Next, we find the starting seed of the first void by finding the first pixel of label value " 0 ". Once the starting seed is found, it is marked as " 3 " and the region keeps on growing until there is no more pixels within the connected region that can be regarded as seeds. The following step is to calculate the area of region marked with " 3 ". If the area is smaller than the area threshold value, it is considered as a small region and will be filled out with object label value (i.e. 1). Otherwise, it will be marked with background label value (i.e. 2). These procedures repeat until no more cavities are detected. We found empirically that an area threshold of about 50 is adequate. The result of this operation is shown in Figure 4.

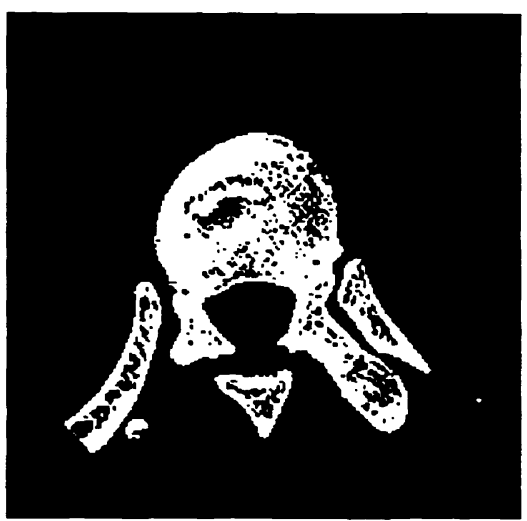

(a) Thresholded image

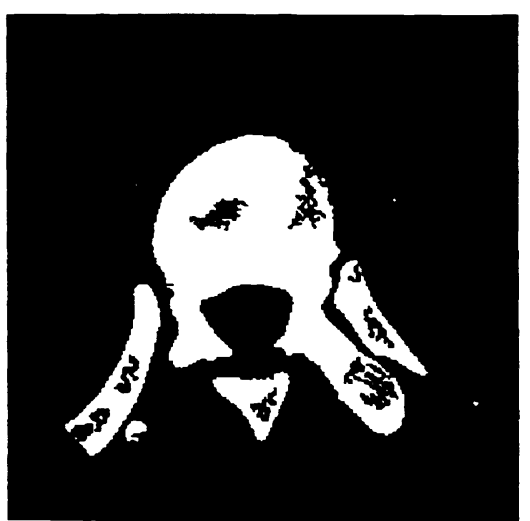

(b) Small voids removed

Figure 5. Result of applying the small void removal algorithm.

\subsection{Binary Morphological Boundary Detector}

After obtaining the desired object regions in an image, the boundaries can be identified by a binary morphological boundary detector. The pixels on the boundary of an object are those that have at least one neighbour that belongs to the background. They can be extracted by binary erosion with a simple structuring element. Erosion ${ }^{11}$, also known as thinning, is defined as a transformation $\mathbf{C}=\mathbf{A} \boldsymbol{\Theta} \mathbf{B}$, which combines sets $\mathbf{A}$ and $\mathbf{B}$ in $\mathrm{N}$-space $\mathbf{R}^{\mathrm{N}}$ following

$$
C=\left\{c \in R^{N}: c=a-b, a \in A, b \in B\right\}
$$

B is called the structuring element of the erosion. A structuring element must be predefined before a morphological operation. A morphological boundary detector can be designed using the fact that erosion by a simple structuring element removes the boundary pixels. This means that the boundary can be stripped away using erosion. Subtracting the eroded image from the original image gives the boundary. The operation essentially "peels away" from set A an outer layer of elements, determined by the size and shape of the structuring element. This detector is defined as:

$$
\text { Boundary }=A-(A \Theta \text { simple })
$$

where A - input binary image, 
simple - structuring element in the form of matrix $\left[\begin{array}{lll}1 & 1 & 1 \\ 1 & 1 & 1 \\ 1 & 1 & 1\end{array}\right]$,

$\theta \quad$ - erode left by right

Figure 6 shows the result of applying this method to extract the boundaries of the image of Figure 5(b).

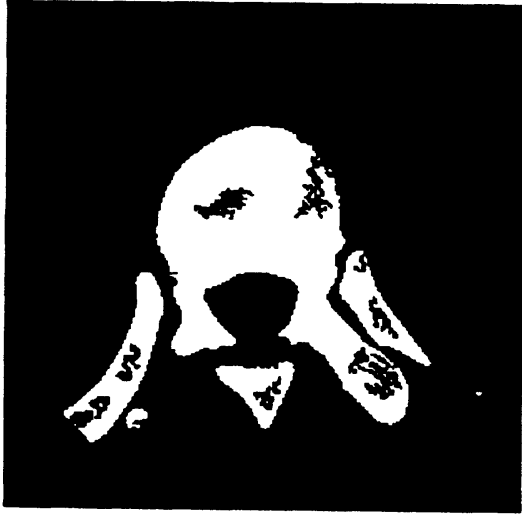

(a) Image processed by small void removal algorithm.

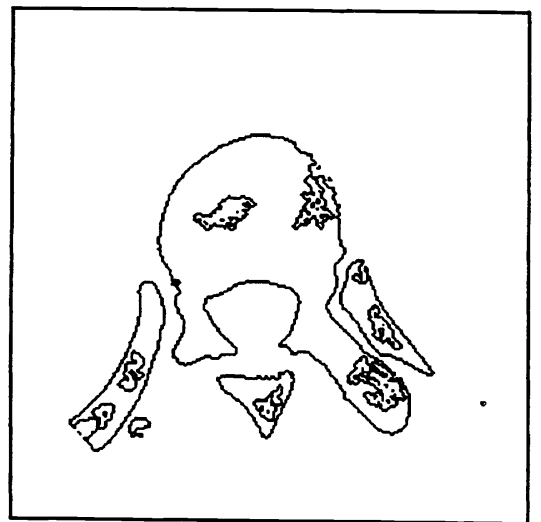

(b) Contour of (a).

Figure 6. Results of boundary detection.

\subsection{Knowledge-based Artifact Removal Algorithm}

The boundary detection procedure described in the previous section generates contours which represent the desired tissue as well as other undesired structures. The undesired structures can be artifacts or noise. Noise usually has a small size, in the

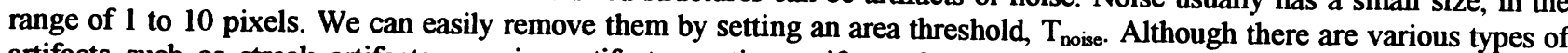
artifacts such as streak artifacts, cupping artifacts, motion artifacts, foreign body artifacts, etc., most of them can be eliminated or reduced significantly by the post-processing algorithms of the CT scanners except the foreign body type. Thus in our study, the primary concern is the artifact caused by the foreign, artificial objects. For example, the containers holding the specimen during scanning possess similar grey levels as the target object. An instance, depicting this, is shown in Figure 7(a). In order to remove this type of artifact, it must be recognized first. The proposed approach uses knowledge-based rules associated with anatomical knowledge. Spinal structures seldom have long, narrow, elongated shapes in their cross-sections. If such a shape is found, it can be safely regarded as artifact. A rule for this generic shape makes use of the length of the object skeleton and the area of the object. A skeleton ${ }^{12}$ is the medial axis or line of symmetry of an object. Since the skeleton of a long, thin object occupies a large proportion of the area of that object, a threshold $T_{\text {artifact }}$ can be selected to identify artifacts. Rules for identifying noise and artifact are set out as follows:

Rule 1: IF

$$
\begin{aligned}
& \text { Area of object }<T_{\text {noise }} \\
& \text { THEN } \\
& \text { Object is noise }
\end{aligned}
$$

Rule 2: $\quad$ IF

$$
\frac{\text { length of object skeleton }}{\text { THEN }}>\mathrm{T}_{\text {artifact }}
$$

Object is artifact

After identifying the artifact, we can delete the contour representing it. There is no theoretical threshold value for artifact. By investigating a set of ten images having the same artifact, an empirical value of 0.5 is chosen. The selection of an optimal threshold remains under investigation. 


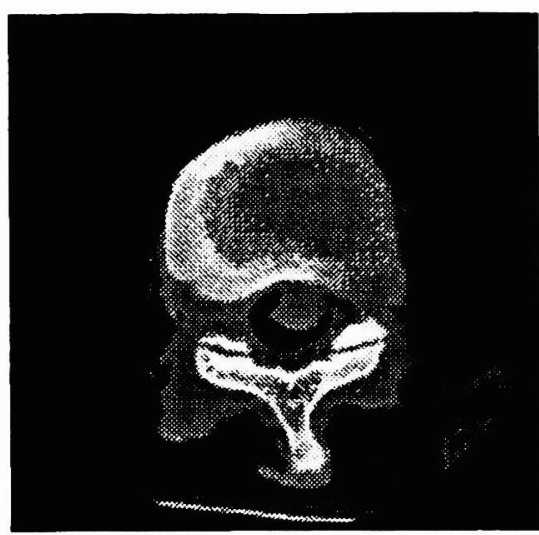

(a)

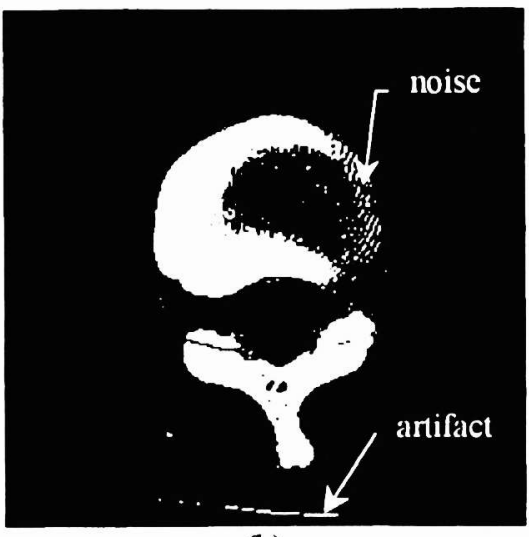

(b)

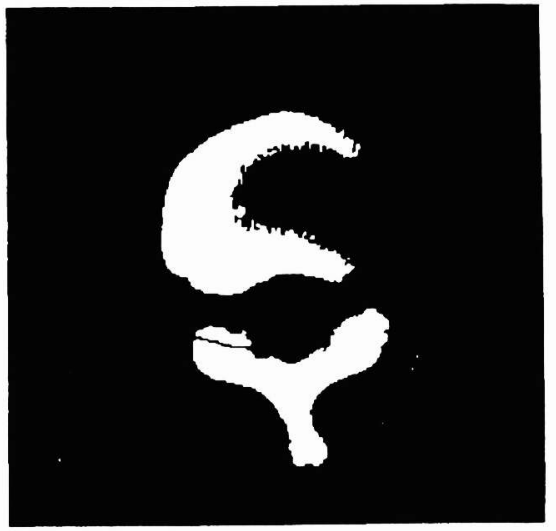

(c)

Figure 7. (a) Original image. (b)Segmented image with some artifacts at the bottom. (c) Artifacts and noise removed

\subsection{Boundary Rectification Algorithm}

Although boundaries are found and artifacts are eliminated by the algorithms reported in the previous sections, the boundaries are raw and bound to contain errors. The errors include incorrect position of extracted edge pixels due to noise, under-segmentation or over-segmentation. Thus we need to identify the erroneous pixels first. Nonetheless, it is very difficult to differentiate between erroneous and correct edge pixels as there is no obvious difference between them. The example shown in Figure 8(a) has four incorrect boundaries. The original piece is a single entity without the indicated small gaps. In order to rectify the boundary, the gaps should be eliminated and linked at its opening. To circumvent this issue, $a$ priori knowledge or edge information from the original image is needed. The knowledge can be divided into two types: macroscopic and microscopic. Macroscopic knowledge involves spatial relationship between contours, characteristic shape of a specific contour, etc. The microscopic level refers to the relations of neighbourhood for each edge pixel. The relations can be intensity gradient and edge strength ${ }^{13}$ in the original image. Adopting both of them, we propose a novel knowledgebased rectification algorithm which considers the topology of every regional skeleton and the Euclidean distance of the skeleton endpoints. It consists of two parts. The first part is to identify the erroneous edge segments by locating their corresponding start and end points. The second part forms new proper edge segments to replace the erroneous ones. In this stage, the suggested proper edge segments is simply a straight line. This may not be very accurate in some situations but is a good alternative in the absence of further information.

The algorithm starts with the generation of the skeleton for every contour. Since the skeleton may not touch the contour, extrapolation with the use of skeleton end pixels and their neighbours is performed in order to extend the skeleton to touch the contour. Between each pair of skeleton end points, the Euclidean distance, $d_{E}$, and the travelling distance along the skeleton, $d_{T}$, are calculated to find out the suspected erroneous edge segments. The erroneous edge segments can be determined by the following two criteria:

1. The ratio of $\frac{d_{E}}{d_{T}}<T_{R}$, where $T_{R}$ is the rectification threshold value.

2. The linear path between each pair of skeleton end points must not intersect any interior part of the region

If a pair of skeleton end points satisfy both criteria, then a false edge segment exists between the end points. After determining the false edge segment, the original edge segment is deleted and a straight-line segment is introduced. From the data sets available, the rectification threshold, $T_{R}$, is determined empirically as 0.1. Figure 8(b) shows the four edge segments rectified by this algorithm. 


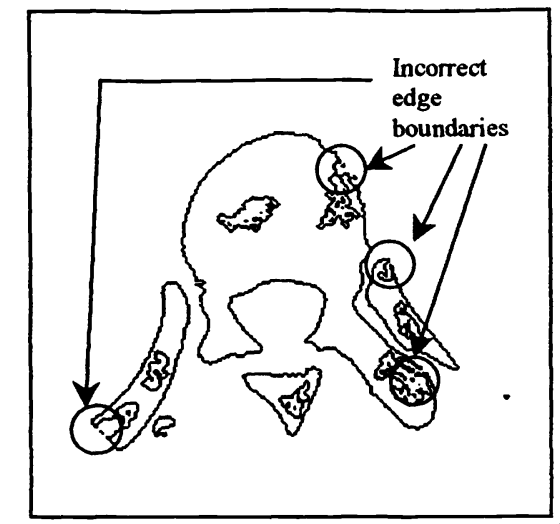

(a) Contour image with incorrect boundaries

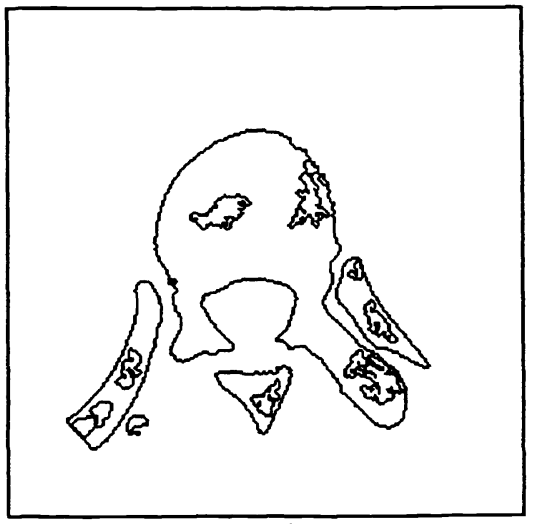

(b) Rectified contour

Figure 8. Results of boundary rectification.

\section{RESULTS}

20 different CT scans acquired on a CT scanner using the "bone" setting were used to test the proposed approach. The image size is $512 \times 512$ pixels with 8 bits per pixel.

The results of these test runs are encouraging. The total processing time for each scan using a Pentium-II $233 \mathrm{MHz}$ system is 10-15 minutes. The most time-consuming operation is Fuzzy Multilevel Thresholding which takes about $60 \%$ of the total processing time for each image. The resulting contours in white are superimposed on the grey-scale CT images as shown in Figure 9. Figure 9(a) shows a thoracic vertebra section. It is observed that the white contours lie more or less on the actual boundary of the bony tissue. Figure 9(b) shows a lumbar vertebra section. It is noted that the gap between the superior articular process and inferior articular process on the right is mistaken as bony tissue. The reason is that the intervertebral disc possesses an intensity range between bone and soft tissue.

The Fuzzy Multilevel Thresholding operation generated thresholds which were consistently adequate. The small void removal and artifact removal algorithms successfully removed small holes and possible artifacts respectively. The boundary rectification algorithm effectively corrected erroneous boundaries.

In all the 20 cases, the bone contour was detected correctly in a large majority of slices. Incidental thresholding problems were invariably related to imperfections in the original image and presence of very narrow gaps. For example, in Figure 9(b), part of the intervertebral disc is misidentified as bony tissue. Also, one of the gaps between the inferior articular process and the superior articular process is fused into a single contour. Severe partial volume effects near these regions also complicated the segmentation task.

For some of the test images with small bony portions (near the ends of a vertebra), bone fragments were misclassified as artifacts and removed. Other errors are encountered incidentally, such as the inability to remove artifacts that are connected to bony tissues.

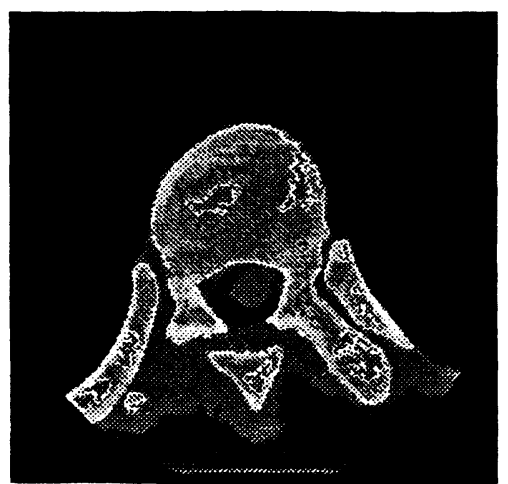

(a) Thoracic vertebra section

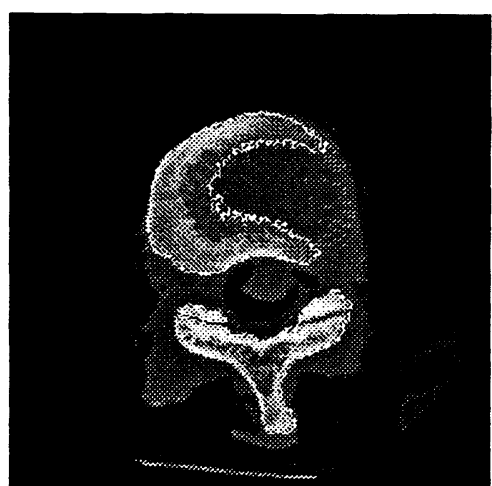

(b) Lumbar vertebra section

Figure 9. Final contours of bony tissue for two of the CT scans tried. 


\section{DISCUSSION}

The approach presented in this paper is dedicated to bony tissue. An interactive editing tool would be a useful supplement. Most of the boundary extraction errors discussed in the previous section can be reduced or eliminated by refining the proposed algorithms, such as employing better definition of artifacts, introducing more knowledge in the knowledge base, and improving the knowledge representation scheme.

Although the algorithms are specific to bony tissue, the key concepts presented in this paper can be extended to other tissues. The grey-level based fuzzy multilevel thresholding is an effective tool for automatic segmentation of other structures. The knowledge-based artifact removal algorithm has the potential of eliminating more types of artifacts if more domain knowledge are included in the knowledge base. For the knowledge-based boundary rectification process, it has a great potential to improve the boundary accuracy if more erroneous boundaries are defined.

\section{CONCLUSION}

We wish to conclude with some remarks about the accuracy of the detected contours. The results presented in this paper have been evaluated by a CT expert and were found to be promising. The visual inspection is a sufficient method to determine the correctness of the detected contours. An alternative is to let CT experts interactively mark the features of interest and compare the results by Pratt's evaluator ${ }^{14}$.

This work highlights some difficulties involved in reliable boundary extraction and rectification of CT images. It calls for techniques that are tolerant of the noise and artifacts present. The contours found by our algorithms are generated by global intensity thresholds which exploit the histogram information. The thresholding are less sensitive to local noise. The contour locations may not be optimal due to non-removable noise and artifacts in some cases. Research on reliable algorithms for modification of complex contours to better correspond with actual edge locations is in a beginning stage.

One particular benefit of this work is that it creates a closed outer contour, making the CT slices more suitable for conversion into surface or solid models.

\section{ACKNOWLEDGEMENTS}

We are indebted to Dr. William Lu from the Department of Orthopaedic Surgery at the University of Hong Kong for his valuable help and comments. We also wish to thank Prof. Wilfred Peh and the Department of Diagnostic Radiology at the University of Hong Kong for their support in obtaining the CT scan data.

\section{REFERENCES}

1. J. D. Boissonnat, and B. Geiger, "Three dimensional reconstruction of complex shapes based on the Delaunay triangulation", Research Report 1697, INRIA, 1992.

2. M. Kass, A. Witkin, and D. Terzopoulous. "Snakes: Active contour models", Int. J. Comput. Vision 1, no. 4, pp. 321$331,1987$.

3. W. C. Chen, N. A. Thacker, and P. I. Rockett, "Adaptive Step Edge Model for Self-consistent Training of Neural Network for Probabilistic Edge Labelling”, IEE J. Vision Image Signal Processing 143, no.1, pp.41-50, 1996.

4. K. H. L. Ho, and N. Ohnishi. "FEDGE - Fuzzy Edge Detection by Fuzzy Catergorization and Classification of Edges", Proceedings of the 1995 International Joint Conference on Artificial Intelligence, pp. 182-199, 1995.

5. W. A. Barrett, and E. N. Mortensen, "Interactive live-wire boundary extraction", Med. Image Anal. 1, issue 4, pp. 331341, 1998.

6. P. Clarysse, F. Poupon, B. Barbier, and I. E. Magnin, " 3 D boundary extraction of the left ventricle by a deformable model with a priori information", Proceedings of 1995 International Conference on Image Processing, vol. 2, pp. 492495, 1995.

7. Xiang-Yong Cheng, A. Ohya, M. Natori, and M. Nakajima, "Boundary extraction method for three dimensional ultrasonic echo imaging using fuzzy reasoning and relaxation techniques", Proceedings of IEEE Conference of Nuclear Science Symposium and Medical Imaging Record, vol. 3, pp. 1610-1614, 1993.

8. I. A. Hunter, J. J. Soraghan, and T. McDonagh, "Fully automatic left ventricular boundary extraction in echocardiographic images", Proceedings of the $22^{\text {nd }}$ annual Computers in Cardiology conference, pp. 741 -744, 1995.

9. A. Deluca, and S. Termini, "A definition of a non-probabilistic entropy in the setting of fuzzy set theory", Inf. Contr. 20, pp. 301-312, 1972.

10. Liang-Kai Huang and Mao-Jiun J. Wang, "Image thresholding by minimizing the measures of fuzziness", Patt. Rec. 28, no. 1, pp. 41-51, 1995. 
11. R. M. Haralick, S. R. Sternberg and X. Zhuang, "Image analysis using mathematical morphology", IEEE Trans. on Patt. Anal. and Mach. Intell. 9, pp. 532-550, 1987.

12. H. Blum, "A transformation for extracting new descriptors of shape", Symposium Models for Speech and Visual Form, Weiant Whaten-Dunn (Ed), Cambridge, MA: MIT Press, 1967.

13. Tony Lindeberg, "Edge Detection and Ridge Detection with Automatic Scale Selection", Proceedings of IEEE Conference on Computer Vision and Pattern Recognition 96', pp. 465-470, 1996.

14. W. K. Pratt, Digital Image Processing, pp.538-543, $2^{\text {nd }}$ edition, John Wiley \& Sons, 1991. 